\title{
Le scritture professionali di Amelia Andreassi: gli ego-documenti di una insegnante italiana del Novecento
}

Os escritos profissionais de Amelia Andreassi: os ego-documentos de uma professora italiana do século XX

Amelia Andreassi's professional writings: the ego-documents of an Italian teacher of the twentieth century

Escritos professsionales de Amelia Andreassi: los ego-documentos de una professora Italiana del siglo $\mathrm{XX}$

\author{
Alberto Barausse \\ Università degli Studi del Molise (Itália) \\ http://orcid.org/0000-0002-8326-046X \\ http://lattes.cnpq.br/5545877149387438 \\ barausse@unimol.it \\ Rossella Andreassi \\ Università degli Studi del Molise (Itália) \\ https://orcid.org/0000-0001-9543-2751 \\ andreassi@unimol.it
}

\section{Riassunto}

Il contributo intende prendere in esame il valore euristico dell'archivio personale di Amelia Andreassi, maestra e poi direttrice di scuole materne private e pubbliche di Bari, importante città dell'Italia meridionale, nel corso del Novecento. La collezione, composta di libri, certificazioni, lettere e materiali didattici, fra cui i quaderni sui quali annotava personali riflessioni sulle pratiche didattiche e ludiche svolte in classe, costituiscono parte del fondo archivistico personale custodito presso il Centro di documentazione e ricerca sulla storia delle istituzioni scolastiche, del libro scolastico e della letteratura per l'infanzia (Ce.S.I.S.) dell'Università del Molise, tra le cui finalità sono previsti il recupero, la conservazione e la valorizzazione degli archivi personali degli insegnanti. Tali fondi permettono una analisi dettagliata della funzione degli scritti personali (egodocumenti) di tipo professionale. Insieme all'uso delle categorie interpretative offerte dalla storia delle culture scolastiche nella analisi si intendono raccogliere le suggestioni proposte dalla storia della memoria scolastica.

Keys words: Archivi personali. Ego-documenti insegnanti. Italia. 


\title{
Resumo
}

A contribuição pretende analisar o valor heurístico do arquivo pessoal de AmeliaAndreassi, que atuou no século XX como professora e diretora de escolas infantil maternais, particulares e estaduais de Bari, importante cidade do Sul da Itália. A coleção composta por livros, atestados, cartas e material didático, entre o qual os cadernos com as suas anotações pessoais sobre as práticas didáticas desenvolvidas na sala de aula, representa parte do acervo pessoal guardado pelo Centro di documentazione e ricerca sulla storia dele istituzioni scolastiche, del libro scolastico e della letteratura per l'infanzia (Ce.S.I.S.) da Universitàdel Molise na recuperação, na conservação e no aprimoramento de arquivos pessoais dos professores. $\mathrm{O}$ acervo permite uma análise detalhada da função dos escritos pessoais (ego-documentos). Juntamente com o uso das categorias interpretativas oferecidas pela história das culturas escolares na análise também pretendemos aproveitar as sugestões oferecidas pela história da memória escolar.

Palavras-chave: Arquivos pessoais. Ego-documentos professores. Itália.

\begin{abstract}
This work analyses a specific collection called "Fondo Amelia Andreassi", preserved at the Ce.S.I.S. (Centre of Documentation and Research on the History of School, School book and Children Literature) of the University of Molise. The collection is composed of documents, books and objects belonged to Mrs. Amelia Andreassi; she was a teacher and school manager who was born in 1909 and died in 2012. The collection's variety reflects the professional figure of a teacher from the past century. The collection starts from the first pedagogical studies of Amelia straight to the certificates of retirement. The fund includes 18 exercise-books; some of them are school reports, others describe the preparation of classes and allow to analyze the methods and pedagogic theories supported by the teacher in that particular historical framework.
\end{abstract}

Keywords: Personal archives. Teacher's ego-documents. Italy.

\section{Resumen}

La contribución analiza el valor heurístico del archivo personal de Amelia Andreassi, quien trabajó en el siglo XX como maestra y directora de guarderías privadas y publicasen Bari, una importante ciudad del sur de Italia. La colección compuesta por libros, certificados, cartas y material didáctico, incluyendo cuadernos con notas personales sobre las prácticas docentes desarrolladas en el aula, representa parte de la colección personal mantenida por el Centro di documentazione e ricerca sulla storia dele istituzioni scolastiche, del libro scolastico e della letteratura perl'infanzia (Ce.S.I.S.) de la Università del Molise en la recuperación, la conservación y el mejoramiento de los archivos personales de los docentes. La colección permite un análisis detallado de la función de los escritos personales (ego-documentos). Junto con la utilización de las categorías interpretativas que ofrece la historia de las culturas escolares, también aprovechamos las sugerencias proporcionadas por la historia de la memoria escolar.

Palabras clave: Archivos personales. Ego-documentos maestros. Italia. 


\section{Introduzione}

Il contributo presenta il caso di studio relativo alle scritture professionali autobiografiche di una insegnante nata all'inizio del secolo scorso che per diversi anni ricoprì anche il ruolo di direttrice didattica. La ricerca si colloca all'interno della cornice storiografica che, in questi ultimi anni, ha offerto molteplici suggestioni provenienti dal rinnovamento storiografico tanto generale quanto specifico nel settore dell'educazione. La ricerca intende collocarsi lungo l'apporto euristico indicato dagli studi orientati a valorizzare la documentazione autobiografica, conosciuta anche come scrittura del sé. Tale ambito di ricerca in Italia ha conosciuto recentemente un notevole sviluppo in riferimento soprattutto a quei fenomeni ed eventi che nel corso del Novecento hanno segnato la vita di milioni di persone. Basterebbe ricordare l'incidenza dei due conflitti bellici mondiali o il fenomeno migratorio, momenti che hanno sollecitato un inarrestabile bisogno di scrittura anche nelle persone di estrazione popolare (Caffarena, 2005; Franzina, 1992; Gabrielli, 2007; Baioni, 2011). All'interno di questo scenario e di questi percorsi della contemporaneità anche sul versante più strettamente storico scolastico si è manifestata l'esigenza di approfondire l'analisi delle forme attraverso cui le scuole, gli insegnanti, gli educatori, il mondo dell'educazione hanno posto il tema della memoria e del suo rapporto con la storia (Vinão, 2005a; Vinão, 2005b; Vinão, 2010, Yanes-Cabrera, Meda, Vinão 2017). A cavallo tra la fine del secolo scorso e il nuovo millennio abbiamo registrato la crescita vertiginosa del fenomeno memorialistico e, parallelamente, degli studi sulla memoria tanto da indurre qualche storico a parlare di «mondializzazione della memoria». Sulla scia dei Memorystudies(Assmann, 1997), anche la memoria scolastica è stata oggetto di un tentativo di chiarimento concettuale molto significativo per offrire agli studiosi un nuovo oggetto di analisi storica fino a parlare di memorie al plurale (Yanes-Cabrera, Meda, Vinão 2017). Molteplici sono, infatti, le forme attraverso le quali la memoria veicola le rappresentazioni della scuola e degli insegnanti. Le più recenti indagini, infatti, ci restituiscono un quadro assai articolato della memoria scolastica, dove accanto alle forme della memoria scolastica collettiva veicolate dalla finzione letteraria e dalla fiction popolare (Ascenzi, 2012), dal teatro e dal cinema (Cohen, 1996, pp.395-420, May\&Ramsland, 2007, pp.135-149, May 2010, pp.623-637), così come quelle trasmesse attraverso le immagini (Del Pozo, 2006; Comas Rubi; Del Pozo, 2018; Sureda; Del Pozo, 2018) o altri media, incontriamo le forme della memoria promossa dalle istituzioni pubbliche nel quadro di ben precise politiche pubbliche della memoria e che si esprimono attraverso la creazione di «luoghi della memoria scolastica» per riprendere una categoria storiografia introdotta da Pierre Nora (1997). Per quanto riguarda la scuola, secondo gli studi di Connercton $(1989,2009)$ la memoria assume forme diverse come i musei o i memoriali della scuola. Ma luoghi della memoria scolastica sono le vie, le piazze e gli edifici scolastici che espongono i nomi degli insegnanti, degli educatori e degli esperti nell'area della pedagogia; l'inaugurazione di targhe, busti e monumenti; il conferimento di decorazioni e distinzioni; la stampa di francobolli e monete commemorative; la celebrazione di funerali promossi dalle associazioni professionali e dalle istituzioni pubbliche in conformità a una memoria specifica (Meda, Pomante, Brunelli, 2019, Sani, Ascenzi, 2016).

Le forme della memoria collettiva e pubblica sono affiancate e arricchite da quelle della memoria scolastica soggettiva espresse attraverso gli ego-documenti -secondo la classificazione di Vinão costituiti dai diari, dalle autobiografie, dalle memorie, ma anche e soprattutto dai ricordi personali recuperati attraverso le testimonianze orali (Vinão, 2005a, pp.19-33).

Il caso di studio delle scritture professionali autobiografiche di Amelia Andreassi incrocia, poi, un altro filone di investigazione recentemente rivalutato dagli storici della scuola, quello relativo alla ricostruzione dei profili biografici degli educatori (Chiosso, Sani, 2013, D’Alessio 2016, Zago 2016 pp.203-234). La ricerca intende far tesoro del ricco filone di studi avviato da Dominique Julia negli anni '90 del '900 che, a partire dalla esigenza di far luce sulle 
culture scolastiche di cui gli insegnanti sono stati espressione, ha prestato una particolare attenzione ai vissuti dei diversi protagonisti del mondo della scuola (Julia, 1996). Un ambito di indagine che ha consentito una forte rivalutazione della ricostruzione dei profili biografici degli educatori prestando considerevole attenzione alla formazione, alla cultura pedagogica e alla condizione dei maestri e delle maestre (Di Pol, 2016, Ghizzoni, 2003, Soldani, 1993, Covato, 1996), nonché ai percorsi d'istruzione riletti nel quadro dei processi culturali che hanno interessato anche gli insegnanti e in maniera più ampia la storia della scuola vista «come parte della più generale storia della vita sociale e dei processi di modernizzazione civile e culturale del paese» (Sani, 2011, pp.358-359). Quello di Amelia Andreassi, analogamente a quello di altri percorsi biografici e professionali di insegnanti cresciuti nell'Italia meridionale nel secolo scorso (D'Alessio, 2017), permette di approfondire, di cogliere maggiori inferenze e di evidenziare le dinamiche sociali, i materiali didattici e le concrete attività d'insegnamento che costituirono l'effettiva cultura scolastica di cui fu portatrice l'insegnante.

La ricostruzione del singolo itinerario di Amelia Andreassi, dal momento formativo all'esercizio professionale, si sviluppa all'interno di un contesto storico, culturale sociale ed economico dell'Italia molto ampio e complesso che copre oltre un secolo di vita dell'insegnante deceduta il 14gennaio 2012 all'età di 102 anni (1909-2012). Amelia Andreassi, infatti, nacque a Napoli il 1 aprile 1909 dall'avvocato Giuseppe, nato nel 1867 e impiegato presso l'ufficio legale del Banco di Napoli, e da Angelina De Mola nata nel 1866 a Fasano in provincia di Bari. La famiglia benestante e con titolo nobiliare di marchesi, subì una gravissima crisi economica e, probabilmente intorno agli anni Venti del Novecento, fu costretta a trasferirsi a Fasano in Puglia ospite di alcuni parenti della famiglia materna. Il padre, in seguito, riuscì a trovare lavoro presso Bari, trasferendosi nel capoluogo pugliese con tutta la famiglia. I coniugi ebbero sei figli di cui Amelia era la quartogenita; ella rimase nubile dedicando la sua vita alla scuola ${ }^{1}$.

\section{Il Ce.S.I.S. dell’Università del Molise e il fondo «Amelia Andreassi»}

I presupposti storiografici e metodologici delineati trovano applicazione nell'analisi empirica del ricco fondo conservato presso il Ce.S.I.S. (Centro di Documentazione e Ricerca sulla Storia delle Istituzioni Scolastiche, del Libro Scolastico e della Letteratura per l'Infanzia) dell'Università del Molise.Nato nel 2006 per promuovere il reperimento e la raccolta di fonti per l'attività di ricerca storico-educativa, come indica lo statuto approvato il 4 maggio 2006, il Centro si propone di promuovere la ricerca, la conservazione e l'utilizzo a scopi scientifici dei documenti inediti e a stampa relativi alla storia delle istituzioni scolastiche ed educative, dell'editoria scolastica e dei libri di testo ed alla storia dell'editoria e della letteratura per l'infanzia in Italia e nel resto d'Europa. Tale finalità viene perseguita attraverso diverse attivitàtra cui l'attivazione e il mantenimento di rapporti e di forme di collaborazione con enti e istituzioni similari in Italia e all'estero; la promozione di convegni, seminari, cicli di conferenze, mostre, pubblicazioni; l'istituzione di borse di studio, cicli di dottorato di ricerca e assegni di ricerca (Barausse, 2020). La struttura oggi, afferisce al Dipartimento di Scienze umanistiche, sociali e della formazione dell'Università del Molise.Molteplici sono i fattori che spiegano la nascita del Ce.S.I.S., tra i quali l'evoluzione della disciplina scientifica nelle università italiane, i mutamenti in seno alla storiografia dell' educazione a livello mondiale e la conseguente internazionalizzazione della ricerca, le problematiche sorte intorno ai luoghi, agli strumenti e alle fonti necessarie per la ricerca nell'ambito storico scolastico ed educativo. Proprio alcuni luoghi privilegiati per la conservazione e trasmissione del sapere e della memoria, come le istituzioni scolastiche, hanno manifestato e ancora sembrerebbero manifestare, i segni di una difficoltà se non addirittura di una evidente noncuranza, nella

\footnotetext{
${ }^{1}$ Le informazioni sulla famiglia di origine e prima infanzia, sono state tratte da documenti privati concessi in visione dagli eredi.
} 
conservazione e promozione del proprio patrimonio, di una preoccupante apatia rispetto al ruolo e alle funzioni storiche esercitate dalla scuola (Barausse, 2010; Soldani, 2006). Bisogna, così, rinviare alle iniziative promosse soprattutto da centri specializzati istituiti per iniziativa di alcuni docenti universitari o di dipartimenti di alcuni atenei, per individuare delle strutture dedicate allo sviluppo della documentazione e della ricerca in questo settore così importante ma così trascurato dalla collettività. L'attenzione posta alla valorizzazione delle fonti prodotte a livello locale, dunque, è stata all'origine dei percorsi di ricerca che il Ce.S.I.S. ha attivato per fare più luce sulle vicende dello sviluppo dei processi di scolarizzazione a livello regionale e delle aree meridionali dell'Italia tra Otto e Novecento. Lo ha fatto sia promuovendo nuovi percorsi di ricerca sia attraverso la scoperta, la conservazione e la valorizzazione di nuove tipologie di fonti documentarie che oggi hanno reso più ampio il patrimonio documentale. In tale prospettiva il Centro di documentazione dal momento della sua nascita ad oggi ha sviluppato una corposa collezione di beni di natura scolastica composta, a fini di ricerca, da documenti librari, archivistici e oggettistica. La raccolta e conservazione del materiale sono stati organizzati rispettando l'attribuzione dei singoli fondi in capo ai loro proprietari-donatori, a ben identificati insegnanti e alunni o in ogni caso ad altrettanto riconoscibili gruppi familiari. Congiuntamente a tali fondi è andato costituendosi, in questi ultimi anni, un «Archivio delle memorie», ossia un fondo costituito da una doppia tipologia di documenti; fotografie che riproducono immagini di vita scolastica a partire dagli ultimi anni dell' 800 fino agli anni '90 del secolo scorso e video interviste ai protagonisti relativi a contesti scolastici di ambito meridionale. All'interno dei diversi fondi conservati dal Ce.S.I.S. si colloca il fondo Amelia Andreassi chesi compone di diverse tipi di materiali. Oltre ai documenti pubblici e privati che, tra l'altro, permettono la ricostruzione della sua biografia formativa e professionale, il fondo è costituito da una collezione libraria fatta di testi utilizzati anche durante gli anni della sua formazione e attività professionale, una collezione di quaderni di lavoro e di cronache scolastiche, i suoi temi di preparazione ai concorsi, le registrazioni vocali delle manifestazioni da direttrice scolastica, le collezioni di materiali scolastici utilizzati a scuola, le fotografie raccolte dalla insegnante. È un fondo che si sta ancora incrementando ed è inventariato e catalogato in forma provvisoria, dato il reperimento ancora in atto di documenti dell'insegnante. I materiali permettono di ricostruire la parabola personale dell'insegnante all'interno del contesto storico in cui si è formata ed è vissuta restituendo preziose informazioni sui percorsi formativi e sulle modalità di insegnamento, soprattutto nella scuola materna della metà del secolo scorso. Il fondo, dunque, si articola in una sezione archivistica e in una sezione libraria. Una parte consistente e interessante del fondo è costituita dai "preziosi" quaderni di cronaca scolastica conservati dalla stessa insegnante con cura e depositati al centro circa due anni prima della sua morte. I quaderni, in tutto 18 , sono classificabili in due tipologie: quelli dove la maestra aveva nel corso degli anni segnato le lezioncine tipo, poesie, stralci di storielle, copioni di recite scolastiche e 6 quaderni di cronache scolastiche relative all'attività professionale svolta tra il 1943ed il1959.Questipresentano una struttura abbastanza omogenea. Nelle pagine inziali sono indicati i contenuti del programma che la maestra intendeva svolgere durante il mese. In quelle successive le pagine indicano quasi quotidianamente le attività e i contenuti svolti e, talvolta, la descrizione dei profili dei bambini che più colpiscono l'insegnante. Le attività fanno riferimento ai contenuti previsti dai programmi suddivisi per materie e soprattutto alla religione, all'educazione morale e fisica, alla lingua italiana, all'educazione intellettuale, al disegno e al canto, recitazione all'aritmetica, alle nozioni varie, esercitazioni dei sensi, vita pratica.

Unica eccezione è il quaderno che raccoglie le cronache dalla maestra dal 1943 al 1957. Si tratta di un quaderno che riporta una selezione delle riflessioni o delle considerazioni riportate negli altri diciassette quaderni, ma la maestra non ci presenta i criteri che l'hanno condotta a tale scelta. Un elemento, questo, da prendere in considerazione per un'analisi critica del documento. Traspare evidente però, anche dal sottotitolo che l'insegnante ha attribuito al 
quaderno, la volontà di soffermarsi soprattutto sui profili dei bambini, quasi a voler rappresentare il quaderno come il frutto di un esercizio professionale costante.

Tutti questi quaderni possono rientrare a buon diritto nelle forme di ego-scrittura su cui la storiografia dell'educazione ha richiamato l'attenzione (Vinão, 2005a).

Figura 1 -La maestra Amelia Andreassi

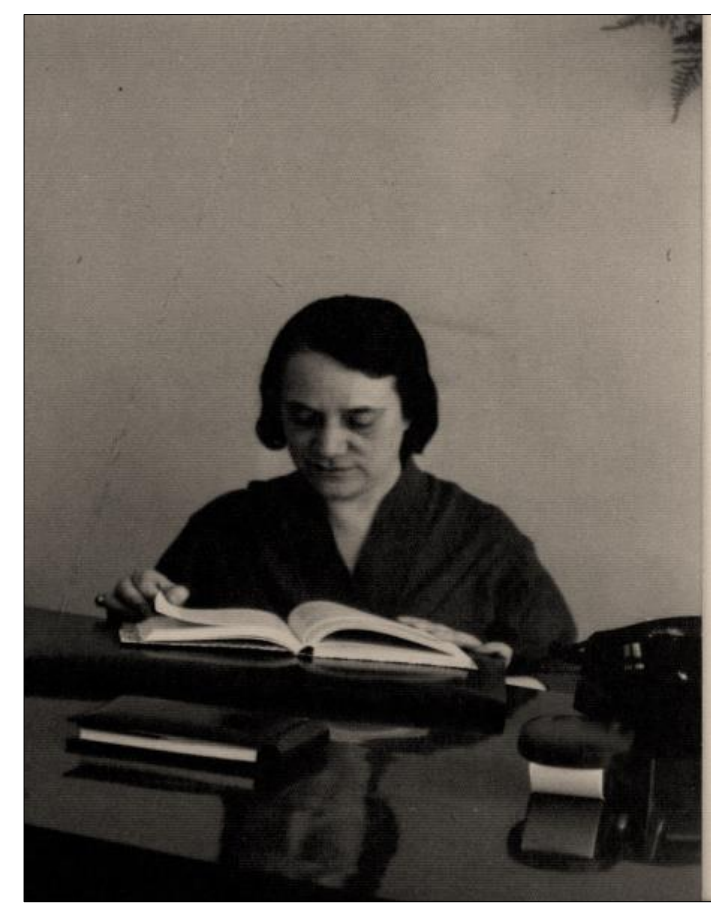

Fonte:Archivio Ce.S.I.S., F. Amelia Andreassi, b. Collezione di materiale fotografico.

\section{Il lungo percorso della maestra Amelia: Le prime esperienze professionali durante gli anni del fascismo.}

Amelia si forma nei primi anni di attuazione della Riforma Gentile. Il filosofo idealista aveva riportato la scuola alla sua strutturazione accentrata e gerarchica cancellando ogni forma di autonomia nell'amministrazione scolastica (Ostenc, 1981, Charnitzky,1996). La riforma aveva ripristinato la scuola elementare ai suoi originari 5 anni con l'aggiunta di un grado preparatorio non obbligatorio per i bambini dai 3 ai 6 anni; inoltre l'obbligo scolastico era stato innalzato fino ai 14 anni. $\mathrm{Fu}$ anche rafforzato il primato culturale e formativo delle scuole classiche e reso più selettivo l'accesso per la prosecuzione degli studi. La riforma, che si basava sul pensiero idealista sostenuto da Gentile e da Codignola, confermava la tradizionale concezione delmaestro come cerniera tra l'élite e le classi popolari che doveva educare, ma rovesciò l'approccio secondo il quale l'insegnante doveva essere dotato di una cultura rudimentale ed elementare per predisporlo alla funzione attraverso una cultura più profonda e adeguata (De Fort 1984, pp. 528-576) e per questo l'istituto magistrale fu elevato al livello degli studi umanistici distanziandosi da quelli professionali. L'ordinamento dell'istituto magistrale prevedeva una durata di sette anni con un grado inferiore di quattro anni e uno superiore di tre anni ed era stato inserito in tutti e sette gli anni lo studio obbligatorio del latino. Inoltre fu eliminata la divisione tra scuole femminili e maschili e furono introdotte come materie di studio il latino e la filosofia insieme alla pedagogia con indirizzo storico-filosofico. Anche l'accesso alla scuola magistrale avveniva attraverso un esame di ammissione che così, per la sua difficoltà, 
sanciva un criterio più selettivo della futura classe docente costringendo gli allievi a una più elevata preparazione di partenza. Fu abolito lo studio della psicologia e anche le discipline professionalizzanti come calligrafia, lavori donneschi, agraria, lavoro manuale e il tirocinio didattico, non senza tensioni tra lo stesso gruppo idealista: la soppressione del tirocinio, come è noto, fu una scelta di Gentile in contrasto con la volontà di Codignola che, invece, lo riteneva determinante per l'apprendimento della professione, tanto che negli anni '30 ci sarebbe stato un ripensamento sull'utilità del tirocinio per la preparazione didattica dei maestri elementari (Di Pol, 2003).

Come è noto il riordino dell'istruzione elementare e la nascita degli istituti magistrali furono accompagnati anche dalla introduzione degli asili infantili, chiamati «scuola materna» (art. 1, R.D. 31 dicembre 1923, n. 3106), come primo grado, definito preparatorio, dell'istruzione primaria. L'ordinamento della scuola materna era di tre anni ma la sua istituzione non fu resa obbligatoria; in questo periodo venne rafforzata anche l'idea che gli asili avessero compiti di custodia ricreativa per i bambini delle classi socio culturalmente più svantaggiate. Il regima fascista non riuscì comunque a inglobare pienamente la scuola materna nei suoi progetti educativi lasciandola relegata a un ruolo caritativo-assistenziale. Per quanto ci fu un tentativo di articolazione curriculare, la scuola materna, in questa fase storica, rimase all'interno di logiche privatistiche e confessionali. Con Bottai, nel 1937, ci fu una riflessione sulla statalizzazione delle scuole materne, e nella Carta della scuola, si prevedeva l'istituzione della scuola materna obbligatoria per tutti i bambini dai quattro ai sei anni finalizzata a disciplinare «le prime manifestazioni dell'intelligenza e del carattere». Non si ottennero però risultati concreti a causa dei grandissimi costi che un'operazione di tal genere avrebbe causato allo Stato(Catarsi, 1994).

All'interno di tale assetto istituzionale determinato dalla riforma Gentile si formò la futura maestra Amelia. Il suo avvicinamento alla professione insegnante e la sua introduzione alle materie pedagogiche avvenne presso l'Istituto Magistrale di Bari dove poté avvalersi del contributo di docenti di spicco tra i quali il noto pedagogista Giovanni Modugno (Andreassi 2013, vol 2, pp.179-180). I documenti in possesso del Centro attestano la sua iscrizione al corso inferiore dell'Istituto Magistrale di Bari nell'anno scolastico 1925-26 per la classe seconda (risulta iscritta per la seconda volta) e alla classe quarta nell'anno 1927-28 ${ }^{2}$. Non sono presenti documentazioni attestanti l'iscrizione al primo e terzo anno e si sta cercando di risalire alle motivazioni di questo vuoto. Nel luglio del 1928 Amelia conseguì il diploma di ammissione al corso superiore dell'Istituto Magistrale. Non abbiamo evidenze certe circa la conclusione dei suoi studi ma dalle fonti in possesso risulta che l'aspirante insegnante riuscì a concludere anche il corso superiore della scuola magistrale.

La presenza dei temi svolti in preparazione al concorso per maestra elementare risalenti al 1931-32 (i compiti di preparazione con un'insegnante privata sono in numero 30) e, soprattutto, un appunto autobiografico, rivelano che vinse il concorso con un tema dal titolo $\grave{E}$ impossibile educare l'infanzia senza possederne la confidenza e l'amore ${ }^{3}$. Nel fondo sono presenti anche i temi, senza data, inerenti la preparazione per il concorso di maestra di scuola materna. Dall'elenco di titoli presentati per il concorso a direttrice scolastica si evince come ella avesse conseguito l'abilitazione a maestra di grado preparatorio (con prova pratica di tirocinio) e il diploma di abilitazione all'insegnamento elementare ${ }^{4}$.

Per divenire insegnante di scuola materna la Riforma Gentile, aveva previsto il possesso del titolo legale di abilitazione all'insegnamento del grado preparatorio conseguito o da conseguirsi presso i corsi estivi triennali previsti dall'articolo 18 della legge del 1913, o in altri

\footnotetext{
${ }^{2}$ Attestati presenti in Archivio Ce.S.I.S., F. Amelia Andreassi, b. Corrispondenza privata e carte personali.

${ }^{3}$ I temi sono raccolti in Archivio Ce.S.I.S., F. Amelia Andreassi, b. Corrispondenza e carte professionali.

${ }^{4}$ Domanda di partecipazione al Sindaco di Bari del 26 ottobre 1959, in Archivio .Ce.S.I.S., F. Amelia Andreassi, b. Corrispondenza e carte professionali.
} 
non meglio precisati corsi o nelle «Scuole di Metodo per l'educazione materna» di durata triennale, istituiti in misura ridotta dalla riforma (Di Pol, 2003). Queste scuole, ritenute professionalmente e culturalmente inferiori, e nelle quali era prevista la presenza obbligatoria dell'insegnamento religioso, nel 1933 furono trasformate definitivamente in scuole magistrali.

Il modello pedagogico che animò prevalentemente le scuole destinate a formare le maestre delle scuole materne fu quello che trasse ispirazione dal metodo introdotto dalle sorelle Agazzi piuttosto che da quello elaborato dalla Montessori. Come è noto dopo l'iniziale sostegno offerto alla pedagogista marchigiana dal fascismo, che ne aveva apprezzato la funzionalità per un apprendimento precoce nel processo di alfabetizzazione, emerse con maggiore evidenza l'incompatibilità tra l'ideologia autoritaria del regime e il modello montessoriano fondato sui principi di libertà e creatività. Ciò permise il sopravvento in Italia del metodo agazziano definito anche da Lombardo Radice «Metodo italiano. Genuino modesto ma geniale e chiaro» che riconosceva in esso le matrici froebeliane e dell'educatore Ferrante Aporti; il metodo agazziano fu molto sostenuto anche dal mondo cattolico. E proprio il modello di maestra di scuola materna, soprattutto nelle Scuole di Metodo, legato alla pedagogia agazziana di «madre pensosa» (Agazzi,1951, Agazzi, Grazzini 2006) più che quello montessoriano di «persona colta e civile», «educatrice di professione» sembra animare l'insegnante pugliese. Amelia Andreassi sembra essere permeata dai modelli educativi e dai riferimenti attinti al modello della pedagogia agazziana sostenuti da un forte spirito cattolico: l'insegnante riporta nella pratica quotidiana il concetto di «maestra come buona mamma» studiando però continuamente e aggiornandosi (ciò si evince dalla raccolta libraria). Su queste basi di fondo innesterà anche altri stimoli di natura più montessoriana.

La sua carriera magistrale iniziò a Bari presso la Scuola elementare statale «Gironda» nell'anno scolastico 1933-34 dove vi rimase fino al 1936. A seguire, nel 1937, ebbe un'esperienza presso Poggiardo in provincia di Lecce, sede che le fu assegnata come provvisoria prima dell'invio, come sede definitiva, a Morciano di Leuca. Alcune missive private ci riportano anche le difficoltà della giovane insegnante nell'alloggiare fuori casa nonostante fosse ospite di una famiglia referenziata ${ }^{5}$.

Il momento decisivo per la sua vita privata e professionale è legato alla inaugurazione nel 1937 a Bari della Scuola materna «Vincenzo Diomede Fresa». L'edificio, collocato nel centro storico di Bari in pieno stile fascista fu eretto dai coniugi Diomede Fresa in memoria del figlio Vincenzo morto prematuramente in modo tragico e fu donato al Comune di Bari con finalità scolastica.

Amelia Andreassi fu inserita a partire dall'anno scolastico 1939-40 come insegnante della scuola materna ma ebbe anche funzioni speciali di «cassiera» e persona di fiducia della famiglia Diomede Fresa che mantenne comunque il controllo della scuola come si legge nell'atto di Convenzione del 1938:

la quale insegnante oltre all'adempimento dei servizi didattici [...] vigilerà, in rappresentanza dei donanti, per l'adempimento delle condizioni relative alla manutenzione e conservazione dello stabile e dell'arredamento, provvederà all'amministrazione dei fondi per elargizioni che pervenissero all'istituto per essere erogate a beneficio delle scolaresche, ed eserciterà le funzioni di Segreteria del patronato ${ }^{6}$.

\footnotetext{
${ }^{5}$ Archivio .Ce.S.I.S., F. Amelia Andreassi, b. Corrispondenza privata

${ }^{6} \mathrm{Si}$ veda in proposito l'atto di Convenzione del 1938, in Archivio .Ce.S.I.S., F. Amelia Andreassi, b. Corrispondenza e Carte professionali
} 
La maestra in questo periodo fu coinvolta anche nelle attività per la fascistizzazione dei giovani. Sin dal 1934, infatti, assunse la responsabilità della locale organizzazione delle "piccole italiane" dell'Opera nazionale balilla?

\section{Dalla fine della seconda guerra mondiale agli anni Sessanta: attraverso i quaderni della maestra}

Dopo la caduta del regime e l'inizio della occupazione degli alleati Amelia è coinvolta nella ripresa delle attività didattiche della scuola dove è costretta a misurarsi con un clima diverso da quello degli anni precedenti, come ci rivelano le sue scritture personali. Il primo giorno di scuolatorna costantemente nelle cronache annuali della maestra. Ma quello dell'ottobre del 1943 colpisce per la rappresentazione di sé-della propria identità- che la maestra vuol dare, quella dell'educatrice cristiana immersa in una vera e propria missione. Dopo pochi mesi dalla caduta del fascismo e dopo poche settimane dalla firma dell'armistizio che porterà l'Italia ad accettare la resa incondizionata alle forze alleate e al disimpegno dalla alleanza con la Germania nazista, risuonano quasi programmatiche le parole con cui la maestra esprime il forte impegno personale come educatrice. Così, il 2 ottobre del 1943 la maestra annota:

Siamo al primo giorno dell'anno scolastico! Anche quest'anno 65 sono i piccoli affidati alle mie cure; sono 65 bimbi del popolo ma diversi, come tutti gli altri bimbi, per educazione, inclinazioni, temperamenti. Ognuno ha una propria personalità e, quindi, deve essere oggetto di uno studio speciale da parte mia. So bene che l'insegnante deve cercare di conquistare l'animo di ciascun alunno per poter frenare i troppo vivaci, spronare i timidi, indirizzare sulla retta via i più baldanzosi e da tutti ottenere quella confidenza che è indispensabile per riuscire nella difficile arte di bene educare. Con l'aiuto di Dio spero di riuscire ad adempiere il non facile compito che da me si richiede quale educatrice cristiana ${ }^{8}$.

Colpiscono i numerosi profili dei bambini che la maestra, animata da un forte spirito di osservazione, tratteggia con ricchezza di particolari. I suoi appunti sembrano, da un lato, voler fissare i loro comportamenti e le loro caratteristiche al fine, poi, di verificarne l'evoluzione nel tempo. Nella stesura dei profili dei bambini, tuttavia, si percepisce il lento mutamento del contesto che si riflette nel cambiamento del linguaggio e delle forme espressive utilizzate per spiegare determinati comportamenti. Nelle cronache del '43 è possibile individuare forme espressive ancora direttamente riconducibili ai cliché propagandistici del regime:

Una vera sorpresa mi ha riserbato quest'anno, il piccolo Bux Gaetano. È ancora, si, un diavoletto di vivacità straordinaria capace di commettere anche qualche monelleria, ma quanta diversità dal Bux dell'anno scorso! L'ho fatto sedere molto avanti in classe, appunto per evitare qualche tiro birbone della sua esuberante vivacità ed, in complesso, lo vedo con quei suoi grandi occhi fissarmi attentamente quando narro qualche storiella o faccio qualche lezione. Mi ha anche colpito il grande desiderio che mostra questo bambino nel voler far parte della guardia d'onore giù al portone quando c'è qualche visita alla

\footnotetext{
${ }^{7} \mathrm{Si}$ veda il certificato rilasciato il 1 maggio 1937 del presidente della organizzazione femminile di Bari, in Archivio Ce.S.I.S., F. A. Andreassi, b. Corrispondenza e carte professionali.

${ }^{8}$ Quaderno delle Cronache della scuola dal 1943 al 1957. 2 Ottobre, Archivio Ce.S.I.S., F. A. Andreassi, b. Collezione Scritture professionali.
} 
scuola. Il suo sogno è quello di maneggiare il fucile. È vero, dunque, che nel bimbo vi è tutto l'uomo in potenza perché in questo bimbo, che oggi è un figlio della lupa, vi è un nuovo guerriero della nostra razza che lo fa già sentire un piccolo soldato capace d'imbracciare il fucile 9 .

E interessante, in proposito, notare le correzioni che la stessa insegnante introdusse non sappiamo se in un periodo successivo- volte a cancellare i riferimenti più evidenti al fascismo e a sostituirli con espressioni più neutre, quasi a evidenziare il disagio della insegnante nell'utilizzare un linguaggio ed un lessico che avevano segnato buona parte della esperienza iniziale: «il grande desiderio che mostra questo bambino di essere soldato e ciò mi dimostra sempre più che nel bimbo vi è tutto l'uomo in potenza. Chi sa se il desiderio di questo mio piccolo allievo non si realizzerà allo sbocciare della giovinezza!» ${ }^{10}$.

Nelle prime pagine del quaderno che fa riferimento agli ultimi mesi del ' 43 si avvertono i riflessi della guerra nei comportamenti dei bambini:

Il piccolo Cassano è uno di quei fanciulli pieni di vita, d'iniziativa e d'intelligenza sveglissima ma si fa soprattutto amare per lo spirito patriottico che lo anima. Mio dice spesso che, quando sarà grande, il babbo gli comprerà 'un cannone' col quale ucciderà tutti i nemici dell'Italia. Il bimbo in parola fa parte della guardia d'onore della scuola ed è felicissimo allorché gli si dice di prendere il moschetto ${ }^{11}$

L'evento bellico insieme allo spirito nazionalistico riaccende anche quello patriottico destinato a ravvivare il coinvolgimento dell'infanzia nelle forme della mobilitazione civile come era già accaduto durante la prima guerra mondiale. La lezioncina sulla lotta allo spreco sembrava aver suscitato unasignificativa partecipazione da parte dei bambini e delle loro famiglie che portarono a scuola "ogni sorta di cose":

Bottiglie, stracci, carte, ferri hanno fatto un po' in classe bella mostra di se, prima di essere raccolti e portati dagli stessi bimbi al posto di raccolta [...] Quanto spirito di patriottismo v'è nel nostro popolo. Quanti sacrifici anche i piccoli possono fare per la nostra Patria in armi! Far comprendere ai bimbi tutta la bellezza del donare non solo ciò che ci è di superfluo ma ciò che soprattutto costa qualche rinunzia ed impone qualche sacrificio, ecco il doveroso e patriottico compito dell'educatrice nell'ora presente ${ }^{12}$.

Anche il natale del 1943 diventava occasione per richiamare il senso patriottico espresso attraverso l'afflato religioso della preghiera personale che l'insegnante rivolgeva a Dio affinché potesse stendere la sua «mano pietosa su tutta l'umanità» e giungesse la «pace con giustizia che tutti chiedono» insieme alla «radiosa vittoria per la nostra Patria» ${ }^{13}$.

\footnotetext{
${ }^{9}$ Quaderno delle Cronache della scuola dal 1943 al 1957.10 novembre. Archivio Ce.S.I.S., F. A. Andreassi, b. Collezione Scritture professionali.

10 Quaderno delle Cronache della scuola dal 1943 al 1957. 10 novembre 1943. Archivio Ce.S.I.S., F. A.Andreassi, b. Collezione Scritture professionali.

11 Quaderno delle Cronache della scuola dal 1943 al 1957. 25 novembre 1943. Archivio Ce.S.I.S., F. A.Andreassi, b. Collezione Scritture professionali.

12 Quaderno delle Cronache della scuola dal 1943 al 1957. 28 novembre 1943. Archivio Ce.S.I.S., F. A.Andreassi, b. Collezione Scritture professionali.

13 Quaderno delle Cronache della scuola dal 1943 al 1957. 30 novembre 1943. Archivio Ce.S.I.S., F. A.Andreassi, b. Collezione Scritture professionali.
} 
Gli eventi bellici costrinsero l'asilo alla chiusura e solo nel gennaio del 1947 fu possibile ripartire. La maestra tornava a scrivere le sue cronache lasciando trasparire un forte sollievo e la personale sofferenza per essere stata lontana dagli alunni: «È passata la bufera della guerra! La scuola si è riaperta dopo anni di abbandono forzato da parte dei piccoli alunni e nostra. Lasciai i bimbi con la speranza di presto rivederli, ed invece sono trascorsi anni!» ${ }^{14}$.

C'è una cosa che colpisce l'insegnante: la presenza di tante mamme vestite a lutto ( «in gramaglia»): «Sono i lutti seminati dall'immane guerra, -annota - tante creature sono prive delle carezze dello loro babbo». Tra gli alunni ci sono, in particolare, cinque orfani di guerra che toccano in modo particolare la sensibilità dell'insegnante: «[...] sarò con questi piccoli più materna che mai cercherò di circondarli di quell'affetto e di quelle cure che a casa più non hanno $[\ldots] \gg{ }^{15}$.

La presentazione dei profili psicologici di molti bambini è una scrittura riflessiva esercitata con costanza anche nel corso degli anni successivi: si percepiscono in questo tipo di scrittura tutti gli studi di pedagogia e filosofia che l'ex alunna di magistrale usa e riporta come conoscenze acquisite e interiorizzate. Vi è nei profili, spesso, una descrizione fisica, un'attenzione allo sguardo e al comportamento generale del bambino. Presente anche un'attenzione psicologica ai diversi tipi di bambini. Viene presentato il bimbo timido, quello irrequieto, il diavoletto, il bravo, l'eccellente, etc. Vengono sottolineati più volte la crescita e il cambiamento anche caratteriale dei bambini da un anno all'altro:

Oggi ho avuto la "gioia" di fare una grande scoperta, Nicola Lanave, che ritenevo un bimbo solo capace di farmi inquietare per le sue troppe monellerie, ha dato, su mia richiesta, saggio della sua bravura. Il bimbo in parola è un vero incorreggibile diavoletto, ha gli occhi piccoli ma di una vivacità eccezionale; io l'ho definito "occhio di topolino". È poi di una irrequietezza incredibile ed è incapace di stare, sia pure per pochi minuti, tranquillo ${ }^{16}$.

Il racconto continua con lo stupore dell'insegnante davanti alla capacità del bimbo di ripetere per la prima volta a memoria delle poesie da lei insegnate durante l'anno. E ancora nel marzo del 1955 scrive:

Zaccaro Vincenzo è un bimbo oltremodo strano. Frequenta la scuola materna da 3 anni, ma mi ha dato sempre gran da fare. Ha un carattere volubile e instabile al cento per cento. Un giorno lo vedi diligente, ubbidiente, educato, l'indomani ribelle ad ogni richiamo e ad ogni esortazione. Cerco sempre di prenderlo con buoni modi $[\ldots]^{17}$.

Nei suoi appunti di cronaca la maestra delinea i profili dei suoi piccoli alunni soprattutto quando i mutamenti nei loro comportamenti colpiscono l'insegnante. Così nel quaderno del 1953-54 la maestra annota con una certa sorpresa il cambiamento di Anna Ardito una bambina che l'anno precedente era sembrata «una bimba insignificante, una di quelle che sono quasi il 'peso morto della scuola'. Quale sorpresa ho avuta, quest'anno, nel rivederla! Intelligenza sveglissima, attiva e servizievole sempre, educata e gentile in tutte le occasioni».

\footnotetext{
${ }^{14}$ Quaderno delle Cronache della scuola dal 1943 al 1957. 30 novembre 1947. Archivio Ce.S.I.S., F. A. Andreassi, b. Collezione Scritture professionali.

${ }^{15}$ Quaderno delle Cronache della scuola dal 1943 al 1957. 30 novembre 1947. Archivio Ce.S.I.S., F. A. Andreassi, b. Collezione Scritture professionali.

${ }^{16}$ Cronaca autografa 1955 in Archivio .Ce.S.I.S., F. A. Andreassi, b. Collezione di scritture professionali.

${ }^{17}$ Ibid.
} 
Ciò che aveva colpito la maestra era soprattutto l'attenzione prestata nei confronti di un bambino da poco entrato nella scuola e rispetto al quale aveva espresso e rivelato «uno spirito materno veramente commovente». Di fronte alle reazioni di pianto del bambino, «inconsolabile per il distacco della mamma» la bambina lo aveva «preso in grembo e l'ha cullato così dolcemente, che il bimbo si è acquietato e per lunghi giorni la buona Anna, è stata l'amorosissima guida e mammina del piccolissimo Nicola» ${ }^{18}$. L'atteggiamento materno, dunque, è qualcosa che caratterizzava anche il comportamento delle bambine.

Tra i profili degli alunni l'insegnante non dimentica di annotare anche quelli di coloro che, a suo avviso, meritano di essere segnalati a strutture più specialistiche come l'Istituto di Psicologia dell'Università di Bari che nel 1950 «chiede la collaborazione della scuola materna per la segnalazione di bimbi anormali del carattere e dell'intelligenza». L'insegnante si sofferma a descrivere e annotare i bambini che ritiene «anormali»per «irrequietezza, scontrosità, instabilità, incapacità alla concentrazione»; descrive bambini anormali nell' aspetto con testa «oblunga e figlio di mamma tubercolotica»; c'è anche il caso del bimbo con «difetto di pronuncia molto spiccato, ma di intelligenza sveglissima e molto irrequieto», viene anche descritto un bimbo come «super-dotato» di «intelligenza sveglissima, padronanza assoluta di sé e delle proprie azioni, intuito spiccatissimo» ${ }^{19}$.

Figura 2 - I bambini della scuola materna Diomede Freda

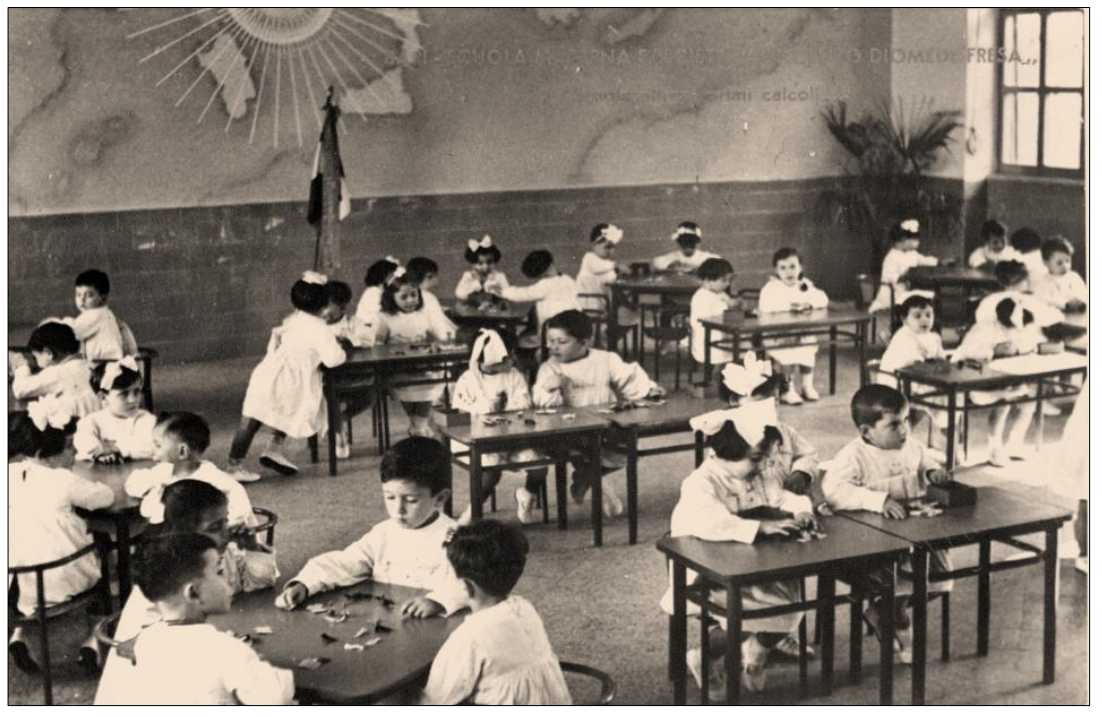

Fonte:Archivio Ce.S.I.S., F. Amelia Andreassi, b. Collezione di materiale fotografico.

Dalle cronache si evincono molteplici tracce intorno alle pratiche utilizzate dalla insegnante nelle sue classi miste per sesso ed età dei bambini come previsto normalmente per le scuole materne (tranne in un solo caso quando la Dirigente nel novembre del 1949 impose la creazione di una classe composta solo dai bimbi di 3 anni con rammarico da parte dell'insegnante Amelia che perse 10 piccoli alunni già ben inseriti).

Considerevole è il controllo che la maestra ha dei numerosi bambini che variano negli anni da un minimo di 42 a un massimo di 86 iscritti. La maestra descrive che sono posizionati in banchetti e in file e spesso sposta i bimbi più vivaci nelle prime file e cerca di coinvolgerli, motivarli e tenerli sotto controllo anche nei loro momenti di intemperanza. Le scolarette più buone e brave sono invece collocate nei tavoli rotondi. Regolare anche l'utilizzo da parte della

\footnotetext{
${ }^{18}$ Quaderno delle Cronache della scuola 1953-54. 30 ottobre 1953. Sunti di cronaca. Spunti di cronaca. Archivio Ce.S.I.S, F. A. Andreassi, b. Collezione Scritture professionali.

${ }^{19}$ Quaderno delle Cronache della scuola dal 1943 al 1957. 8giugno 1950. Archivio Ce.S.I.S., F. A. Andreassi, b. Collezione Scritture professionali.
} 
maestra delle bambine più grandi indicate come "piccole mamme" per supportare il suo lavoro di accoglienza dei più piccoli.Ma tra i temi ricorrenti che si incontrano nelle Cronache della maestra troviamo la preoccupazione per la numerosità della sua scuola, della sua classe. Una problematica legata al forte squilibrio sociale tra il Sud e le altre aree dell'Italia che determinava una evidente scarsità di strutture educative per la prima infanzia nelle aree meridionali del Paese e la speranza di vedere accrescere l'offerta di scuole materne. La grave situazione post bellica impose al governo di concentrarsi maggiormente sull'istruzione elementare lasciando interamente l'iniziativa della scuola materna agli enti locali e privati.Questo è uno dei passi esemplificativi che illustra la riflessione della maestra intorno alla condizione emergenziale in cui viveva la scuola materna barese di fronte alla esplosione della domanda di scuole materne verso la metà degli anni ' 50 a cui le esistenti scuole materne, non riuscivano a dare risposta:

quest'anno 86 iscritti!... è scoraggiante ormai la posizione della scuola materna nella nostra Bari. Quando sarà placato questo implacabile bisogno di scuole della nostra popolazione? È inutile dire che una simile condizione incide, in modo deleterio, sull'animo delle educatrici tutte. Esse vedono con sgomento le enormi difficoltà e responsabilità che ogni anno sono costrette ad affrontare. Si dice che la speranza sia l'ultima a morire e noi tutte speriamo ardentemente, che un giorno non lontano, verrà la sospirata riscoss a della scuola materna del mezzogiorno finora tanto negletta e nuove scuole sorgeranno per colmare le molteplici lacune ${ }^{20}$.

L'attenzione alle problematiche materiali presentate dalla scuola è accompagnata anche dalla esigenza di una costante e forte affermazione dell'identità della scuola, un processo che accompagna la costruzione delle stesse singole identità dei piccoli alunni. Le scritture personali della insegnante non omettono di indicare gli annuali momenticommemorativi della fondazione della scuola, occasione per manifestare tra i bambini il sentimento di riconoscenza verso la famiglia benefattrice e il valore della propria scuola attraverso un discorso di elogio verso la famiglia Diomede Fresa: «Ho cercato di inculcare nell' animo dei bimbi, il rispetto per questo edificio sorto da un grande dolore per il bene dei figli del popolo» ${ }^{21}$. Sono espressioni semplici che, però, rivelano il radicamento di una concezione dell'istruzione, largamente diffusa tra gli insegnanti italiani, orientata a fareuna distinzione netta tra gli itinerari destinati alle classi popolari, come la scuola materna e la scuola elementare, e quella per le classi dirigenti (Sani, 2011).

Non si incontrano nelle scritture della maestra commenti specifici relativi all'attuazione dei nuovi programmi per la scuola materna ed elementare predisposti dalla sottocommissione all'istruzione del Governo Militare composta anche da Carleton Washburne (allievo di John Dewey) e che divennero attuativi nel 1945-46. Come è noto i nuovi programmi erano ispirati all'attivismo pedagogico americano e miravano a creare una scuola non impostata sul nozionismo e sull'ascolto passivo degli insegnanti, ma una scuola attenta alla psicologia dell'alunno più che a quella del maestro. Più che sulla trasmissione di nozioni, ritenute fini a sé stesse in quanto mutevoli, le pratiche didattiche erano destinate a sviluppare la ricerca e lo sviluppo delle capacità critiche attraverso l'esperienza diretta. I programmi ispirati a questa nuova pedagogia si ponevano l'obiettivo non solo di continuare a combattere l'analfabetismo ma anche - e soprattutto- di rendere più solida la cultura eticocivile,mirando alla costruzione di un modello di cittadinanza responsabile e democratica. Le pratiche

\footnotetext{
${ }^{20}$ Quaderno delle Cronache della scuola dal 1943 al 1957. inizio anno 1951-52 in Archivio Ce.S.I.S., F. Amelia Andreassi, b. Collezione di scritture professionali.

${ }^{21}$ Quaderno delle Cronache della scuola 195758.26 ottobre 1957. in Archivio Ce.S.I.S., F. Amelia Andreassi, b. Collezione di scritture professionali
} 
didattiche seguite dall'insegnante riflettono parzialmente il nuovo spirito democratico e ruotano intorno a due assi fondamentali. Da un lato venivano prevalentemente esercitate le attività di carattere ludico e, dall'altro, la maestra svolgeva delle brevi e succinte "lezioncine" i cui contenuti tendevano a sviluppare alcune conoscenze relative alla religione, all'educazione morale e fisica, alla lingua italiana; all'educazione intellettuale, all'aritmetica, alle nozioni varie facendo largo uso difiabe, raccontini, del disegno e del canto, della recitazione. Lo svolgimento delle attività prevedeva un ampio spazio ai giochi e ai piccoli lavori. Si trattava di coniugare sin dalla scuola dell'infanzia i momenti ludici, che potevano esser tanto liberi, quanto individuali e collettivi ed imitativi alle prime forme di occupazione «riposanti» svolte attraverso l'uso di «bastoncini e gettoni». Attraverso tali momenti educativi la maestra non intendeva solo conoscere i bambini e promuovere la loro socialità, ma anche favorire l'apprendimento di determinati comportamenti come il rispetto dell'ordine e della pulizia, la promozione delle capacità di osservazione, di concentrazione, di riflessione. L'attività di Amelia Andreassi come educatrice intendeva riflettere, così, a partire dal gioco il comportamento di una madre che avesse compreso i compiti formativi:

Conoscendo bene [...] il grande ausilio che è il gioco per la maestra di scuola materna, specie nei primi giorni di scuola, faccio eseguire molti giochetti fra bambini vecchi e nuovi per poter studiare, specie in questi ultimi, il carattere, le tendenze e le attività di ciascuno. Anch'io come una buona mamma mi propongo di seguire molto da vicino ciascun alunno affidato alle mie cure ${ }^{22}$.

Le pratiche ludico-didattiche erano, poi, contraddistinte da frequenti narrazioni di raccontini e di favole che talvolta accompagnavano le brevi e sintetiche lezioni. Nei diari cronaca dell'insegnante troviamo frequenti riferimenti alla narrazione delle favole moraleggianti come quella de Il corvo e la volpe (Cronaca del 16 novembre 1953) - su cui la maestra appuntava «morale per i bimbi: vanità punita»- o quelle di Cappuccetto rosso (Cronaca del 15 novembre 1955) piuttosto che quella di Biancaneve (Cronaca del 30 novembre 1955) o di Pinocchio (Cronaca del novembre 1955). Amelia attingeva dalla personale collezione di testi sia di carattere ottocentesco che novecentesco come $\mathrm{La}$ Bella addormentata nel bosco, La Fata giusta dei fratelli Grimm (Bologna, C.E.L.I.), le letture di Pietro Thouar o il Mondo piccino. Racconti dell'amica dei bimbi, (Milano, Fratelli Treves, 1881) o il deamicisiano Libro Cuore, i romanzi di Giuseppe Fanciulli (Fiore romanzo per ragazzi, Firenze, Bemporad, 1930) e la raccolta dei testi di Anna Foce autrice di una serie di volumetti largamente diffusi e usati nella seconda metà del novecento dalle insegnanti di scuola materna ${ }^{23}$. O ancora di Ginevra Pellizzari La fatina Gocciadoro, (Milano: Piccoli), Tredicino, (Milano: Boschi), Alice nel paese delle meraviglie (Milano: Boschi), Fanciullezza in marcia di M.R. Berardi, (S.A.S., Torino) Nino e Ghita, il segreto di Carolina, L’avventura di Carolina, editi dalla milanese Risi.

\footnotetext{
${ }^{22}$ Quaderno delle Cronache della scuola dal 1943 al 1957. 28 novembre 1958. Archivio Ce.S.I.S., F. Andreassi, b. Collezione Scritture professionali.

${ }^{23}$ Tra i volumi pubblicati all'indomani della seconda guerra mondiale e che risultano presenti nella biblioteca dell'insegnante si segnalano quelli di Foce, A.Giochiamo. Duecento e più ricreazioni per i bimbi della scuola materna. Brescia: La Scuola, 1940; Ead. Gli animali e i bimbi. Racconti di animali per i bambini della scuola materna e delle prime classi elementari. Brescia: La Scuola, 1949; Ead. Con Gesù. Raccolta di racconti per l'educazione religiosa dei bambini di scuola materna e delle prime classi elementari. Brescia: La Scuola 1949; Ead., Lavorare con gioia. Raccolta di raccontini che si riferiscono alla vita pratica dei bambini di scuola materna e delle prime classi elementari. Brescia: La scuola, 1949; Ead., VI. Gli animali e i bimbi. Brescia: La Scuola, 1949; Ead. VII. C'era una volta. Raccolta di fiabe, novelle, racconti diversi per i bambini di scuola materna e delle prime classi elementari. Brescia: La Scuola, 1949; Ead., Piccolo mondo. Raccolta di racconti in riferimento agli esercizi per l'educazione linguistica e sensoriale dei bambini di scuola materna e delle prime classi elementari. Brescia: La Scuola, 1949; Ead., La voce del cuore. Raccolta di racconti per l'educazione morale e patriottica dei bambini di scuola materna e delle prime classi elementari. Brescia: La Scuola, 1949.
} 
Il canto, il disegno e la recitazione costituivano, invece, altrettante numerose occasioni per far esprimere la spontaneità dei bambini.

Una pratica costante era quella dedicata all'insegnamento linguistico per il quale i diari rivelano l'attenzione prestata dalla maestra al confronto fra il dialetto e la lingua al fine di favorire la sostituzione di termini dialettali con quelli italiani; la correzione dei difetti di pronuncia, le conversazioni su soggetti diversi anche non presenti nell'aula e l'esatta pronunzia del proprio nome e cognome. Ma le pratiche dell'insegnante, poi, intendevano focalizzare l'attenzione sui nomi e sull'articolo facendo leva anche su giochi associati all'identità (il nome dei bambini, il nome della scuola, il nome della direttrice) o a esercizi di lingua parlata come quando, ad esempio, mostrava ai bambini vari oggetti e disegnini e faceva premettere la parola adatta. «Noto che questo esercizio è utilissimo per far parlare i bimbi. Anche i più timidi hanno fatto sentire la loro vocina» ${ }^{24}$.

Nelle pratiche didattiche ed educative della maestra ricopre un ruolo significativo l'educazione religiosa. Questa occupa una parte importante nell'azione quasi quotidiana della maestra. I temi religiosi trattati in diversi modi permeano tutto l'insegnamento di Amelia. La sua adesione personale alla fede cristiana, e in particolare al cattolicesimo, diventa motivo di ispirazione continuo nella promozione di iniziative e pratiche educative di stampo religioso. Le feste, le commemorazioni sono lo spunto per introdurre argomenti di carattere religioso. Così lo sono la festa di Ognissanti per spiegare «come e perché sono diventati Santi» insieme ai «Nomi dei Santi più conosciuti dai bimbi. Nostro sforzo per imitarli in questa vita, per poi raggiungerli in Paradiso ${ }^{25}$. Il 4 novembre, dopo la festa dei defunti, i bambini rientrati a scuola sono sollecitati dalla insegnante a raccontare come hanno passato la commemorazione ma è l'occasione per la preghiera e spiegare il contegno da tenere in chiesa. E così anche la festa della Immacolata concezione, e poi quella della annunciazione della vergine e, nel mese di maggio, l'attenzione alle celebrazioni mariane. Questi momenti religiosi avrebbero accompagnato quelli più importanti dell'anno quali il Natale, con la festa del presepe vivente, e la Pasqua. A gennaio sono i re magi e l'epifania ad essere l'occasione per insegnare la preghiera del Padre nostro. Ma accanto alle festività della nascita e della morte di Gesù la maestra non omette di collocare nei mesi di febbraio, la commemorazione «con semplici parole» della conciliazione fra stato e chiesa. Inoltre un altro appuntamento importante per introdurre i momenti di educazione religiosa è quello legato alla festa del santo locale. In particolare per i bambini della scuola barese sono la figura e la festa di San Nicola ad essere occasione per raccontare la storia del santo. Una tradizione antica, quella di presentare la storia dei santi. Le giornate invernali, soprattutto quelle più fredde e piovose sono l'occasione anche per spiegare ai bambini $\mathrm{i}$ «sentimenti di amore e di pietà verso i più poveri, i vecchi e gli ammalati» cercando di far comprendere «ciò che si potrebbe fare per lenire le loro pene e far contento Gesù» ${ }^{26}$.

All'interno di questo contesto, tuttavia, alcune annotazioni della maestra sono significative perché esprimono con evidenza la presenza di forti segnali di secolarizzazione in corso nella società barese, riflesso di quanto accade più in generale nei contesti urbani italiani a partire dagli anni Cinquanta del Novecento e che investono con sempre maggiore evidenza anche i comportamenti dell'infanzia a partire da quella prescolare. C'è una costante che torna all'inizio di ogni anno scolastico. L'esigenza di

\footnotetext{
${ }^{24}$ Quaderno delle Cronache 1957. 26 novembre 1953. Archivio Ce.S.I.S., F. A. Andreassi, b. Collezione Scritture professionali.

${ }^{25}$ Quaderno delle Cronache 1953- 1954. 29 ottobre 1953. Archivio Ce.S.I.S., F.A.Andreassi, b. Collezione Scritture professionali.

${ }^{26}$ Quaderno delle Cronache 1953- 1954. 11 gennaio 1954. Archivio Ce.S.I.S., F. A. Andreassi, b. Collezione Scritture professionali.
} 
dover insegnare il segno della croce. In tutti i quaderni la maestra, infatti, segnala quasi con le stesse parole, la seguente preoccupazione:

Ogni inizio di anno scolastico, mi tocca fare la solita triste constatazione: quasi nessuno dei bimbi nuovi, sa fare il segno della Croce. Eccomi allora, che con grande pazienza, chiamo vicino uno per volta i piccini, e li esercito nei movimenti della manina e faccio apprendere le parole del "Segno del Cristiano" 27.

Ma si veda pure quanto riportato nel quaderno del 1958 quando la maestra scriveva in maniera quasi analoga: «E una amara costatazione che si ripete ogni anno ed è allora che chiamo vicino a me con grande pazienza le 'nuove reclute' e, sostituendomi alla mamma insegno a ciascuno il 'Segno del Cristiano'» ${ }^{28}$.

Anche nel corso degli anni Cinquanta sono ricorrenti le attività finalizzate a infondere gli insegnamenti morali e quelli etico-civili. Questi ultimi si traducono in richiami e momenti soprattutto di carattere patriottico. I diari raccontano, ad esempio, come la maestra colga le date delle commemorazioni civili per spiegare ai bambini il significato delle feste civili come quella del 4 novembre «per parlare della Patria e della bandiera italiana»e insegnare il canto «Alla bandiera» ${ }^{29}$.

Accanto a questi momenti, poi, l'insegnante traeva spunto da alcune vicende legate in modo specifico al contesto politico degli anni Cinquanta per illustrare altri argomenti, come quelli di geografia. Ad esempio la lezioncina per far conoscere i nomi delle città era l'occasione per entrare nelle vicende della questione triestina e nel contenzioso tra Italia e Jugoslavia sulla città:

Come si chiama la città nella quale viviamo? Allora siamo...Come si chiama la nostra Patria? Allora noi siamo... Che forma ha l'Italia? Che cosa è quell'azzurro che la circonda? Qual è la capitale dell'Italia? Quali altri nomi di città e paesi ricordiamo? Qual è la città più cara al nostro cuore? Trieste- Perché? Perchè altri uomini cattivi ce la vogliono levare e prendersela loro. Quanti colori ha la nostra bandiera? Come si chiamano? E la bandiera del Papa quanti colori ha? Come si chiama? Come si chiama il Papa? Chi è? Dove sta? ${ }^{30}$.

Ancora nel quaderno del 1958 al 10 dicembre annota come:

Partendo dal nome della nostra città, ho data questa mattina ai miei scolaretti, l'idea di Patria. Insegnando il nome sacro dell'Italia ne ho mostrato il "ritratto" ed ho detto qual è la Sua capitale e la Sua bandiera ${ }^{31}$.

Le considerazioni della maestra sono spesso inframmezzate anche con altri riferimenti al contesto reale di un'Italia che, uscita dal conflitto mondiale, si avvia a vivere

\footnotetext{
${ }^{27}$ Quaderno delle Cronache 1953- 1954. 14 ottobre 1953. Archivio Ce.S.I.S., F. A. Andreassi, b. Collezione Scritture professionali.

${ }^{28}$ Quaderno delle Cronache 1957- 1958. 22 novembre 1958. Archivio Ce.S.I.S., F. A.Andreassi, b. Collezione Scritture professionali.

${ }^{29}$ Quaderno delle Cronache 1953- 1954. 6 novembre 1953. Archivio Ce.S.I.S., F. A.Andreassi, b. Collezione Scritture professionali.

${ }^{30}$ Quaderno delle Cronache 1953- 1954. S.d.. Archivio Ce.S.I.S., F. A. Andreassi, b. Collezione Scritture professionali.

${ }^{31}$ Quaderno delle Cronache 1958- 1959. 10 dicembre 1958. Archivio Ce.S.I.S., F. A.Andreassi, b. Collezione Scritture professionali.
} 
la fase di transizione verso la grande trasformazione che sarà più evidente nel corso degli anni Sessanta del Novecento. Le cronache della maestra, pertanto, non omettono i riferimenti ad alcune vicende condivise con i bambini, come episodi di cronaca o catastrofi naturali (come lo straripamento del Po nel novembre del '51), le epidemie. L'allagamento del Polesine, una vasta area dell'Italia settentrionale, fu accolta con trepidazione dalla maestra che coinvolse direttamente le famiglie dei bambini al fine di organizzare la raccolta di fondi e vestiario per le popolazioni in difficoltà:

Una immane sciagura si è abbattuta su l'Italia settentrionale. Il Po ha straripato per chilometri e chilometri, tutto travolgendo. Città fiorenti e campagne ubertose sono sommerse dalle acque; Rovigo ed Adria tra i maggiori centri sono i più coliti dalla sciagura. Quante persone hanno dovuto abbandonare case ed averi, quante mamme sono state private delle loro creature e quanti bimbi senza più la carezza materna. Oggi ho parlato ai miei alunni della grande disgrazia dell'Italia del nord e li ho invitati a portare qualche indumento da offrire ai sinistrati. Nei visini attoniti ho letta tutta la comprensione per i fratelli tanto provati dalla sventura, e sono certa che a casa, le loro innocenti voci, sapranno toccare il cuore della mamma che certamente diverrà generosa nel dare ${ }^{32}$.

Un altro momento destinato a rimanere impresso nella memoria collettiva ful 'ultima grave epidemia di poliomielite che colpì l'Italia del Novecento, che determinò un posticipo generalizzato dell'inizio dell'anno scolastico e a Bari cominciò con quaranta giorni di ritardo: «Come ho trepidato in questo periodo di forzata inattività, per tante mamme ansiose per la sorte dei loro piccini!» 33 .

La collezione dei quaderni si ferma all'anno scolastico 1958-59, ma non presenta commenti specifici relativi ai cambiamenti dei programmi intervenuti proprio nel 1958, quando furono emanati gli Orientamenti per l'attività educativa della scuola materna. Tuttavia non doveva essere difficile per l'insegnante accogliere le sollecitazioni rivolte agli insegnanti ad ammettere flessibilità negli orari ma soprattutto a non anticipare l'insegnamento del leggere, dello scrivere e del calcolo e dare prevalenza al gioco e al fare. Altrettanta sicurezza, probabilmente, contribuì a dare la riaffermazione dell'ispirazione religiosa di matrice cattolica dei programmi e quella più decisa dell'educazione morale e sociale del bambino con attenzione all' "amore verso la famiglia, il prossimo e la patria». Temi che l'insegnante aveva, probabilmente, iniziato a recepire attraverso la partecipazione alle forme associative e all'uso di strumenti espressione della presenza dei cattolici nell'istruzione materna, come la sezione locale della Associazione Educatrice Italiana e la lettura di riviste come «Scuola materna»; ma anche attraversola partecipazione alle diverse iniziative promosse in Italia del Centro didattico nazionale per scuola materna (Cdnsm). Nelle cronache dell'insegnante si percepisce questo clima culturale in cui è immersa attraverso l'impegno profuso per l'educazione civile dei bambini, la lotta contro la permanenza delle forme dialettali e i tentativi di incentivare forme di attivismo pedagogico anche se rimase forte, nell'insegnante Amelia, il modello agazziano su cui si era formata.

32 Quaderno delle Cronache della scuola dal 1943 al 1957. 13 novembre 1951. Archivio Ce.S.I.S., F. A.Andreassi, b. Collezione Scritture professionali.

${ }^{33}$ Quaderno delle Cronache 1958- 1959. 20 novembre 1958. Archivio Ce.S.I.S., F. A.Andreassi, b. Collezione Scritture professionali. 
L'insegnante mantenne l'incarico presso la scuola materna fino al 1960, anno in cui vinse il concorso interno per titoli del Comune di Bari per divenire direttrice. Il 1 giugno 1960, infatti, risultò vincitrice al primo posto in graduatoria e prese servizio il 1 ottobre presso la Scuola Comunale Materna «Regina Margherita» del capoluogo pugliese $^{34}$. Nel 1964 chiese il trasferimento come direttrice alla Scuola Materna «Giuseppe Dalfino» per un avvicinamento alla sua residenza.

\section{Conclusioni}

Le vicende e l'esame delle scritture professionali soggettive dell'insegnante Amelia Andreassi, rivelano il potenziale euristico di fonti, quelle che si riferiscono alle scritture e alla letteratura autoreferenziale, generalmente poco considerate in ambito storico-educativo. Tali fonti, esprimono il bisogno di soggettività e rivelano il modo molto personale e introspettivo attraverso il quale il ceto insegnante si misurò con le sfide professionali in un contesto particolarmente complesso come quello rappresentato dalle scuole di Bari, un contesto urbano significativo del Mezzogiorno d'Italia sollecitato da tensioni e squilibri sociali accentuati.

L'incontro tra le esigenze espresse in questi ultimi decenni dalla storia dell'educazione intesa come storia delle culture scolastiche, delle pratiche scolastiche della quotidianità scolastica e la storia delle scritture autobiografiche hanno permesso di attribuire valore anche alle forme di scrittura elaborate in forma soggettiva e personale dagli insegnanti relative al campo professionale e, in particolare, dalle insegnanti. I testi esaminati hanno permesso di scoprire quell'occhio mobile («l'ojo movil») che Vinão ha individuato come il punto di vista personale del soggetto sulla realtà. Le scritture professionali, in questo caso, esprimono una soggettività femminile, quella della maestra sulla realtà scolastica quotidianamente sperimentata. La prospettiva micro-storica dalla quale si è preso le mosse ha consentito, peraltro, di verificare e individuare le specificità attraverso le quali operò la maestra e il suo modo personale di interpretare le prescrizioni e le normative, adattandole alla sua mentalità e cultura magistrale, in «un gioco di relazioni reciproche tra il regno della libertà e quello della necessità» (Vinão, 2000, p. 11).

\section{Bibliografia}

AGAZZI, Aldo.; GRAZZINI M. Sulle fonti del metodo Pasquali-Agazzi e altre questioni. Interpretazioni, testi e nuovi materiali. Brescia: Centro studi pedagogici «Pasquale Agazzi», 2006.

AGAZZI, Aldo. Il metodo delle sorelle Agazzi per la scuola materna. Brescia: La Scuola, 1951.

ANDREASSI, Rossella. Giovanni Modugno. In:Dizionario biografico degli Educatori (1800-2000). Milano: Editrice Bibliografica, 2013, vol. 2, pp. 179-180.

ASCENZI, Anna. Drammi privati e pubbliche virtù. La maestra italiana dell'Ottocento tra narrazione letteraria e cronaca giornalistica. Macerata: Eum, 2012.

ASSMANN, J. La memoria culturale: scrittura, ricordo e identità politica nelle grandi civiltà antiche. Torino: Einaudi, 1997.

\footnotetext{
${ }^{34}$ Graduatoria dei vincitori al concorso del 1960 in Archivio Ce.S.I.S., F. A. Andreassi, b. Corrispondenza e Carte professionali
} 
BAIONI, Massimo. Patria mia. Scritture private nell'Italia unita (a cura di). Bologna: Il Mulino, 2011.

BARAUSSE, Alberto. Alla scoperta di nuovi tesori: le carte e i libri scolastici come beni culturali. In I.Zilli (ed.), Atlante delle emergenze culturali del Molise. Campobasso: Palladino Editore, 2010, pp. 127-144.

BARAUSSE, Alberto. Il «Centro di Documentazione e Ricerca sulla Storia delle Istituzioni scolastiche, del Libro scolastico e della Letteratura per l'Infanzia») dell'Università degli Studi del Molise tra dimensione locale e scenari internazionali della ricerca storico-scolastica ed educativa. in La pratica educativa. Storia, memoria e patrimonio. Atti del 1 Congresso nazionale della Società Italiana per lo studio del Patrimonio Storico-Educativo. Macerata: EUM, 2020, pp. 69-86.

CAFFARENA, Francesco. Lettere dalla Grande Guerra. Scritture del quotidiano, monumenti della memoria, fonti per la storia. Il caso italiano. Milano: Unicopli, 2005.

CATARSI, Enzo. L'asilo e la scuola dell'infanzia. Storia della "scuola materna" e dei suoi programmi dall'Ottocento ai giorni nostri. Firenze: La Nuova Italia, 1994.

CHARNITZKY, Jurgen. Fascismo e scuola. La politica scolastica del regime (1922-1943). Firenze: La Nuova Italia, 1996.

CHIOSSO, Giorgio; SANI, Roberto (Org).Dizionario Biografico dell'Educazione 1800 2000. Milano: Editrice Bibliografica, 2013.

COHEN, S. Postmodernism. The New Cultural History, Film: Resisting Images of Education. Paedagogica Historica. 32(2), 1996, pp. 395-420.

COMAS RUBI, F.; DEL POZO ANDRÉS, M.D.M. Photography, propaganda and education. Historia, Memoria de la Educacion, n.8, 2018, pp. 9-21. DOI: https://doi.org/10.5944/hme.8.2018.22053.

CONNERTON, P. How Societies Remember. Cambridge, Cambridge University Press, 1989.

CONNERTON, P. How Modernity Forgets.Cambridge, Cambridge University Press, 2009.

COVATO, Covato. Un'identità divisa: diventare maestra in Italia tra Otto e Novecento. Roma, Archivio Guido Izzi, 1996.

D’ALESSIO, Michela. Una biografia collettiva degli educatori italiani degli ultimi due secoli. Note a margine del seminario di studi "Educatori e istituzioni scolastiche in Italia: percorsi, bilanci e prospettive d'indagine", Potenza, 26 novembre 2015.History of education \& Children's literature, vol. 11, n.2, 2016.

D'ALESSIO, Michela. La professione docente in Italia meridionale nel primo Novecento. L'esperienza del Maestro Ialenti in Molise (1881-1915). Rivista di storia dell'educazione, $\mathrm{n}$. 2, 2017, pp. 325-340. 
D'ALESSIO, Michela. Il duro calle. Le memorie di un ispettore scolastico italiano (Berengario Galileo Amorosa-1865/1937). Revista Linhas, v.18, n. 36, jan./abr.2017, pp.178-199. DOI: https://doi.org/10.5965/1984723818362017178.

DE FORT, Ester. I maestri elementari italiani dei primi del Novecento alla caduta del fascismo.Nuova Rivista Storica, 68 (4.-5), 1984, pp. 528-576.

DEL POZO ANDRÉS, M.D.M. Imágenes e historia de la educación: construcción, reconstrucción y representación de las prácticas escolares en el aula. História de la Educación, 25, 2006, pp. 291-315.

DI POL, Redi Sante. Cultura pedagogica e professionalità nella formazione del maestro italiano. Dal Risorgimento ai nostri giorni. Torino: Marco Valerio, 2003.

DI POL, Redi Sante. La scuola per tutti gli italiani. L'istruzione di base tra stato e società dal primo Ottocento a oggi. Milano: Mondadori, 2016.

FRANZINA, Emilio. L'immaginario degli emigranti. Miti e raffigurazione dell'espeirenza italiana all'estero fra i due secoli. Paese (TV): Pagus, 1992.

GABRIELLI, Pietro. Scenari di guerra, parole di donne. Diari e memorie nell'Italia della seconda guerra mondiale. Bologna: Il Mulino, 2007.

GHIZZONI, Carla. Il maestro nella scuola elementare italiana dall'Unità alla Grande Guerra, in Roberto Sani e Angelino Tedde (eds.), Maestri e istruzione popolare in Italia tra Otto e Novecento. Interpretazioni, prospettive di ricerca, esperienze in Sardegna. Milano: Vita e Pensiero, 2003, pp. 19-79.

GHIZZONI, Carla. Essere maestri in Italia fra Ottocento e Novecento in E. Becchi e M. Ferrari (eds.), Formare alle professioni. Sacerdoti, principi, educatori. Milano: Franco Angeli, 2009, pp. 454-491.

JULIA, Domenique. Riflessioni sulla recente storiografia dell'educazione in Europa: per una storia comparata delle culture scolastiche, Annali di storia dell'educazione e delle istituzioni scolastiche, vol. 3, n. 3, 1996, pp. 119-147.

MAY, J.; RAMSLAND J., The Disenchantment of Childhood: Exploring the Cultural and Spatial Boundaries of Childhood in Three Australian Feature Films, 1920s1970s.PaedagogicaHistorica, 43(1), 2007, pp. 135-149.

MAY, J. A Field of Desire: Visions of Education in Selected Australian Silent Films.Paedagogica Historica, 46(5), 2010, pp. 623-637.

MEDA, Juri; POMANTE, Luigiaurelio; BRUNELLI, Marta.Memories and public celebrations of education in contemporary times. Presentation. History of Education \& Children's Literature, vol. 14, n.1, 2019, pp. 11-21.

NORA, Pierre. Les Lieux de Memoires. Paris: Gallimard, 1984-1992. 3 voll.

OSTENC, MichelLa scuola italiana durante il fascismo, Bari, Laterza, 1981. 
ROUSSO, H. Face aupassé. Essay sur la memoire contemporaine. Paris: Belin, 2017.

SANI, Roberto. Sub specie educationis: studi e ricerche su istruzione, istruzioni scolastiche e processi culturali e formativi nell'Italia contemporanea. Macerata: EUM, 2011, pp. 358-359.

SANI, Roberto; ASCENZI Anna, Oscuri martiri eroi del dovere. Memoria e celebrazione del maestro elementare attraverso $\mathrm{i}$ necrologi pubblicati sulle riviste didattiche e magistrali nel primo secolo dell'Italia unita (1861-1961). Milano: Angeli, 2016.

SOLDANI, S. Nascita della maestra elementare in Simonetta Soldani e Gabriele Turi (eds.), Fare gli italiani. Scuola e cultura nell'Italia contemporanea I. La nascita dello Stato nazionale. Bologna: il Mulino, 1993, pp. 67-129.

SUREDA GARCIA,B.; DEL POZO ANDRÉS, M. D M. (eds.) Images of the European Child in History of Education \& Children's Literature, vol. 13, n.1, 2018, pp. 11-16.

VINÃO FRAGO A. Relatos y relaciones autobiograficas de profesores y maestros, in A. Escolano Benito e J. M. Hernandez Diaz (edd.), La memoria y el deseo. Cultura de la escuela y educacion deseada, Valencia, Tirant lo Blanch, 2002, pp.135-175.

VIÑAOFRAGO, A. La memoria escolar: restos y huellas, recuerdos y olvidos, Annali di Storia dell'Educazione e delle Istituzioni Scolastiche, n. 12, 2005a, pp. 19-33.

VINÃO FRAGO, A. La Historia de la Educación ante el siglo XXI: tensiones, retos y audiencias. In: FERRAZ LORENZO, M. (Org.), Repensar la historia de la educación: nuevos desafíos, nuevas propuestas, 2005b, pp. 147-166.

VIÑAO FRAGO, A. Memoria, patrimonio y educación. In Educatio Siglo XXI, n. 2, 2010, pp. 17-42.

YANES CABRERA, C.; MEDA, J.; VIÑAO FRAGO, A. (eds.). School Memories. New Trends in the History of Education. Springer: Cham 2017.

ZAGO, G. La biografia nella storiografia e nella storiografia dell'educazione. Linee evolutive di un rapporto complesso. Espacio, Tiempo y Educatiòn, 3 (1), 2016, pp. 203-234. DOI: https://doi.org/10.14516/ete.2016.003.001.11. 